\title{
Decision making by general practitioners in diagnosis and management of lower urinary tract symptoms in women
}

\author{
Irwin Nazareth, Michael King
}

\begin{abstract}
Objective-To identify factors influencing decision making by general practitioners in the diagnosis and treatment of lower urinary tract symptoms in women. tices.

Setting-Two suburban London general prac-

Subjects-Women presenting to their family doctor with lower urinary tract symptoms.

Design-After each consultation the doctor completed a questionnaire on presenting symptoms; clinical examination; investigations undertaken; presence of psychological, social, and menstrual problems; patients' requests for antibiotics; antibiotic prescribing; knowledge of the patient; attitude towards the consultation; and any other factors assisting in diagnosis and management. Finally, doctors predicted the presence or absence of clinically important bacteriuria. Each woman completed a demographic questionnaire, the 12 item general health questionnaire, and the modified menstrual distress questionnaire, after which each provided a clean catch midstream urine sample. Case notes were examined for information on previous reports of results of urine analysis.
\end{abstract}

Results-When the general practitioners did not know the patients well they were 4.5 times more likely to assume that there was a clinically important infection. When they knew the patient well, they were four times more likely to make a correct prediction of the test result and 12 times less likely to prescribe antibiotics. Doctors were five times more likely to predict the test result correctly in patients from social classes 1 and 2 and were six times more likely to prescribe antibiotics for the older women in the sample.

Conclusions-In women presenting with urinary tract symptoms, these family practitioners seemed to take no particular regard of physicial, psychological, or menstrual factors in making their assessments. They were most accurate in their prediction of the result of urine analysis and least likely to prescribe antibiotics when they had a good general knowledge of the patient. Which came first, the diagnosis or prescribing, is difficult to say and probably differed in individual cases. Doctors tended to be more conservative in their management of older women and those whom they knew less well.

Department of

Academic Psychiatry, Royal Free Hospital School of Medicine, London NW3 2QG

Michael King, senior lecturer

Correspondence to:

Dr Nazareth.

$B M \mp$ 1993;306:1 103-6 processes in secondary care settings, little work has been conducted in primary care. ${ }^{1}$ Within an average consultation time of 6-10 minutes per patient, general practitioners must treat large numbers of people who consult with a wide range of symptoms. Three principal factors govern diagnosis and management of patients within the general practice consultation. These are the characteristics and training of the doctor, the characteristics of the patients and the symptoms they bring to the consultation, and the process of the consultation itself. ${ }^{2}$ For example, the prescription of antibiotics for sore throats may be influenced by minor variations in the patient's psychological and social history. ${ }^{3}$

Six per cent of women attending general practitioners consult for lower urinary tract symptoms. ${ }^{45}$ How do general practitioners diagnose and manage these complaints? Up to $95 \%$ of doctors may prescribe an antibiotic before receiving the results of urine analysis, ${ }^{\circ}$ even though $50 \%$ of patients will not have a clinically important infection on culture. This is reflected in evidence indicating that family doctors prescribe antibiotics appropriately in only $45 \%$ of cases. ${ }^{8}$ It has been claimed in a recent study, however, that doctors can make accurate diagnoses up to $80 \%$ of the time. ${ }^{9}$ In this British study the doctors involved could not account for their diagnostic precision but the authors of the report presumed that they were able to balance illness factors, such as dysuria, with patient factors, such as psychological and social status, to arrive at a correct diagnosis. To rationalise current clinical practice, much more information is needed on how doctors make clinical decisions.

We aimed to identify those factors in the management of lower urinary tract symptoms in women that assisted general practitioners in making a diagnosis and influenced the prescription of antibiotics.

\section{Method}

The study was conducted in two group general practices in suburban London. Six general practitioners took part. All women aged $16-45$ years presenting over a three month period with symptoms of frequency or dysuria for which no antibiotics had been prescribed during the preceding four weeks were asked by the doctors to participate. Women who were pregnant or had any other concurrent medical disorder were excluded. After each subject had given informed consent, information was collected in the following sequence.

The general practitioner-After each consultation the doctors completed a one page form containing open questions concerning presenting symptoms, clinical examination, and investigations carried out; questions on the presence of psychological, social, and menstrual problems; factors which assisted them in diagnosis and management; visual analogue scales of their knowledge of the patient (from "not at all" through to "very well") and attitude towards the consultation (from "dismayed" through to "pleased"); whether antibiotics had been prescribed and whether the patient had requested 
them; and their prediction of the result of urine analysis as no infection, insignificant bacteriuria, or bacterial infection.

The subject-After giving informed consent to take part, each woman completed a demographic questionnaire concerning age, sex, marital status, and occupation; the 12 item version of the general health questionnaire, a well validated screening questionnaire used to detect probable psychological disorder (recent comparisons show that this short version compares well in terms of validity and reliability with longer versions ${ }^{10}$; the questionnaire avoids symptoms that may be due entirely to the effects of physical illness); and the modified menstrual distress questionnaire (this 34 item questionnaire, adapted for general practice from an earlier version, ${ }^{11-13}$ collects information on physical, psychological, and social aspects of health in the seven days before menstruation in each cycle). ${ }^{11}$

Before leaving the doctor's surgery, each woman gave a clean catch midstream urine sample, after having received written instructions on the correct procedure. Samples were refrigerated and delivered to the pathology service with 24 hours. Clinically important bacteriuria was defined, according to clinical practice, as greater than $10^{5}$ bacterial colonies per litre. ${ }^{14}$

The case notes of each patient were examined to collect information on previous results of urine analysis.

\section{Results}

Sixty one women consulting with lower urinary tract symptoms were asked to take part. Two refused and the general practitioners failed to complete the questionnaires for five. who had agreed. Thus, data on 54 women $(88 \%)$ were analysed.

\section{PATIENTS' RESPONSES}

The mean age of the women was 28 years; nine women were single. Nine were in social classes 1 and 2 and 30 in social classes 3 and 4 . Forty seven (87\%) were born in the United Kingdom (table I).

Fifteen women presenting with urinary symptoms $(28 \%)$ had significant bacterial growth on culture of the midstream urine specimen and will be referred to as the women with positive results. The remaining 39 had negative results on culture or had a report of insignifi-

TABLE I-Demographic information. Figures are numbers (percentages) unless otherwise indicated

\begin{tabular}{|c|c|c|}
\hline & \multicolumn{2}{|c|}{ Results of urine analysis } \\
\hline & Positive & Negative \\
\hline Mean age (years) & $29 \cdot 5$ & $28 \cdot 5$ \\
\hline \multicolumn{3}{|l|}{ Mean age (years) on completion of full time } \\
\hline \multicolumn{3}{|l|}{ Marital state: } \\
\hline Single & $2(13)$ & $7(18)$ \\
\hline Married & $7(47)$ & $20(51)$ \\
\hline Cohabiting & $3(20)$ & $11(28)$ \\
\hline Separated or divorced & $3(20)$ & $1(3)$ \\
\hline \multicolumn{3}{|l|}{ Country of birth: } \\
\hline United Kingdom & $13(87)$ & $34(87)$ \\
\hline Commonwealth & 0 & $4(10)$ \\
\hline Europe & $1(13)$ & $1(3)$ \\
\hline \multicolumn{3}{|l|}{$\begin{array}{l}\text { Social class } \\
\text { Soc }\end{array}$} \\
\hline $1-2$ & $4(27)$ & $5(13)$ \\
\hline $3-4$ & $6(40)$ & $24(62)$ \\
\hline $5-7$ & & $3(8)$ \\
\hline Housewife & $4(27)$ & $6(15)$ \\
\hline Unemployed or student & $1(7)$ & $1(3)$ \\
\hline
\end{tabular}

${ }^{\star}$ Classified according to Goldthorpe and Hope. ${ }^{16}$

TABLE II-Scores on psychological tests and results of urine analysis

\begin{tabular}{lcc}
\hline Results of urine analysis & $\begin{array}{c}\text { General health } \\
\text { questionnaire } \\
\text { score } \geqslant 2\end{array}$ & $\begin{array}{c}\text { Modified menstrual } \\
\text { distress } \\
\text { questionnaire } \\
\text { score } \geqslant 1\end{array}$ \\
\hline Positive $(n=15)$ & 8 & 6 \\
Negative $(n=39)$ & 17 & 16 \\
\hline
\end{tabular}

TABLE III-Presenting symptoms as recorded by the general practitioners. Values are numbers (percentages) of women

\begin{tabular}{lcc}
\hline & \multicolumn{2}{c}{ Results of urine analysis } \\
\cline { 2 - 3 } Symptoms & $\begin{array}{c}\text { Positive } \\
(\mathrm{n}=15)\end{array}$ & $\begin{array}{c}\text { Negative } \\
(\mathrm{n}=39)\end{array}$ \\
\hline Dysuria & $13(87)$ & $30(77)$ \\
Frequency & $13(87)$ & $33(85)$ \\
Other symptoms: & $2(13)$ & $5(13)$ \\
Vaginal symptoms & $1(6)$ & $8(21)$ \\
Abdominal symptoms & $3(20)$ & $10(26)$ \\
Back pain & $5(33)$ & $2(5)^{\star}$ \\
Haematuria & $1(6)$ & 0 \\
Fever & 0 & $7(18)$ \\
Urgency & $1(6)$ & $2(5)$ \\
Pvulvae infection & &
\end{tabular}

*Proportionate difference $=0 \cdot 28,95 \%$ confidence interval $=0 \cdot 034$ to 0.530 , $x^{2}=7 \cdot 96, \mathrm{df}=1, \mathrm{p}<0.01$.

TABLE IV-Prediction of result of urine analysis

\begin{tabular}{lcc}
\hline General practitioner's prediction & $\begin{array}{c}\text { Positive result } \\
(\mathrm{n}=15)\end{array}$ & $\begin{array}{c}\text { Negative result } \\
(\mathrm{n}=39)^{\star}\end{array}$ \\
\hline $\begin{array}{l}\text { None or insignificant infection } \\
\text { Clinically important infection }\end{array}$ & 4 & 18 \\
& 11 & 20 \\
\hline *In one case the doctor omitted to make a prediction. &
\end{tabular}

cant bacteriuria (less than 1000 bacteria/ml urine) and will be referred to as the women with negative results.

Twenty four women had no reports of results of urine analysis in their practice notes. Eleven women had reports of both positive and negative cultures, 18 negative cultures, and one only a positive culture.

Median scores on the general health questionnaire were 2 in the women with positive results and also the women with negative results. Twenty five (47\%) scored above a cut off score of 2 , indicating a degree of emotional disturbance that would benefit from intervention, at least at the level of primary care (table II). The median score on the modified menstrual distress questionnaire was 6 for both groups of women, with 22 $(44 \%)$ women reporting menstrual disturbance as defined by a score of 1 or more. ${ }^{11}$

\section{DOCTORS' RESPONSES}

The doctors indicated in their questionnaires that 43 women had dysuria and 46 increased frequency of micturition. Other, much less common, symptoms were backache, abdominal pain, haematuria, urgency, and vaginal symptoms (table III). The presence of psychological, menstrual, and social disturbances were reported by the general practitioners in only 10,6 , and 12 , respectively, of the 54 patients. Clinical examination and other investigations were seldom undertaken: three women were given an abdominal and vaginal examination and one had a blood test.

The doctors made an accurate prediction of the result of urine analysis in 29 patients, achieving a sensitivity of 0.73 and a specificity of 0.39 (table IV).

The doctor's ability to detect a psychological or social problem was studied by comparing their assessment (as indicated on the doctors' questionnaire) with that of the general health questionnaire. Social problems were included as it may be difficult for doctors to distinguish them from psychological problems in patients who present in primary care with generalised dissatisfaction. The doctors achieved a sensitivity of 0.83 and a specificity of 0.68 in detecting such problems. A similar comparison between general practitioners' recordings of menstrual problems and patients' self reports showed that the doctors detected menstrual problems with a sensitivity of 0.09 and a specificity of 0.86 .

Thirty seven women were prescribed antibiotics. Equal proportions of women with positive and negative results $(67 \%)$ received an antibiotic, reflecting overdiagnosis by doctors in the group with negative results (false positives). Although only 11 women requested antibiotics, there was a trend for more requests to come from women with negative results on urine analysis. 
PREDICTORS

A search was made for those factors which determined whether the doctor prescribed antibiotics; considered that a clinically important infection was present; correctly predicted the result of urine analysis. This approach was based on previous work suggesting that the decision to prescribe often preceded the application of a diagnostic label. ${ }^{3}$

Variables which were predictors at the $p \leqslant 0 \cdot 20$ level were entered in a forward stepwise logistic regression to establish their independent effects. This level of significance was chosen to restrict the numbers of variables entered into the logistic regression analysis to those for which at least a trend was observed. In a sample of this size, this reduces the risk of generating an overoptimistic result with respect to the importance of each variable and goodness of fit. ${ }^{15}$ Continuous variables were dichotomised about their means or medians depending on the distribution of the data.

Prescription of an antibiotic was associated with the doctor's knowledge of the patient (Mann-Whitney $\mathrm{U}=220.5, \mathrm{Z}=-2.49, \mathrm{p}<0.01$; difference between medians $=28,95 \%$ confidence interval 1 to 44$)$. Other predictor variables associated with antibiotic prescribing $(p \geqslant 0.05)$ were increased frequency of micturition, identification of social problems by the general practitioner, a patient's request for antibiotics, and the patient's age. Logistic regression showed that only age and the general practitioner's knowledge of the patient were independent predictors of antibiotic prescription. A doctor well acquainted with the patient was 12 times less likely to prescribe antibiotics $(p=0.002 ; 95 \%$ confidence interval of odds ratio $2 \cdot 4$ to 60 ), and if the woman was older than the mean age of 29 years she was six times more likely to receive antibiotics $(p=0.03 ; 1 \cdot 2$ to $29 \cdot 5$ )

Diagnosis of clinically important infection-The less well the general practitioner knew the patient, the more likely he or she was to diagnose clinically important infection (Mann-Whitney $U=231 \cdot 5$, $\mathrm{Z}=-1.99, \mathrm{p}<0.046$; difference between medians $=18$, $95 \%$ confidence interval 0 to 34 ). Other variables that predicted whether the doctor believed there was clinically important infection $(p \geqslant 0.05)$ were the patient's age, presence of dysuria, identification of psychological problems, and the doctor's attitude to the patient Logistic regression analysis revealed that only the doctor's knowledge of the patient was an independent predictor of whether he or she diagnosed a significant infection. Doctors were 4.5 times more likely to diagnose significant infection when they did not know the patient well $(95 \%$ confidence interval of odds ratio 1.4 to $14.5, \mathrm{p}=0.01$ ).

Correct prediction of the result of urine analysis was significantly associated with the social class of the patient $\left(\chi^{2}=4.15, \mathrm{p}<0.04\right.$; difference in proportions $=0.25,95 \%$ confidence interval 0.01 to 0.48 ) and the general practitioners' knowledge of the patient (Mann-Whitney $\mathrm{U}=168.5, \mathrm{Z}=-2.48, \mathrm{p}<0.013$, difference between medians $=12,0$ to 34 ). Other variables that predicted the doctor's accuracy $(p \geqslant 0.05)$ were the patient's age and a report of increased frequency of micturition. Logistic regression analysis, however, showed that only social class and the doctor's knowledge of the patient were independent predictors of accuracy. General practitioners were five times more likely to make a correct prediction of the result of urine analysis in patients of social classes 1 and $2(p=0.06$; $95 \%$ confidence interval of odds ratio 0.09 to 28.4 ) and four times more likely in those patients with whom they were better acquainted $(p=0.02 ; 1.2$ to 13.5$)$.

\section{Discussion}

The most important influence on decisions reached by these doctors was their general knowledge of the patient as measured on a visual analogue scale. Whether they liked the patient played no part. They were more accurate in their prediction of the result of urine analysis when they knew the patient well, and they erred on the side of predicting infection when they were less familiar with the patient. They were also less likely to prescribe antibiotics if they knew the patient well. Which came first, the diagnosis or prescribing, is difficult to say and probably differed in individual cases. Although we have explored independent predictors of the result of urine analysis and prescribing of antibiotics, the two are unlikely to be autonomous. Howie has shown that prescribing leads diagnostic decisions, ${ }^{3}$ and Hjortdahl and Borchgrevink, in a study of the influence of general practitioners' knowledge about their patients on use of resources, reported that when doctors knew their patients well they were more likely to manage problems expectantly and were less likely to prescribe. ${ }^{17}$

It is impossible to know what component of general knowledge of this type was helpful. A good knowledge of the patient might include knowing whether she had previously presented with symptoms and other features of her physical history. However, the results of previous urine testing did not contribute to diagnosis or prescription of antibiotics. Perhaps just as importantly, knowing a patient well may allow the doctor to be more frank about the need to await the outcome of the culture of the urine sample. The decision making that precedes the prescriptions of antibiotics in general practice is complicated. As in other series we found that about half of the prescriptions were not justified. The prescriber is, however, attempting to balance several disparate influences and decide on what to do for the best. ${ }^{18}$ Doctors may be uncomfortable about their decision to prescribe and require skills in negotiating whether or not the patient's expectation for a prescription should be fulfilled. Our results indicate that doctors are better able to negotiate "rational" prescribing when they know the patient well.

Other factors which influenced doctors' accuracy in prediction and prescription of antibiotics were the social class and age of the patient. The doctors made more accurate predictions in women in social classes 1 and 2, but gave antibiotics more commonly to women who were relatively older. Social class as a predictor was independent of knowledge of the patient and cannot be explained simply on the basis that general practitioners might be on more familiar terms with women from higher social classes. Women in the higher social classes may also be more medically minded and more able to articulate their symptoms than those in lower socioeconomic groups.

O'Dowd et al reported that general practitioners prescribed antibiotics in $80 \%$ of cases that later turned out to have significant infection but only $23 \%$ of cases in which no significant infection was subsequently reported on culture of the urine sample. ${ }^{9}$ Women without infection had more entries in their practice notes for so called psychosomatic disorders, and thus the authors assumed that doctors used these notes, together with the presenting symptoms, to make their decision. The authors made no assessment of the doctors' more general knowledge of their patients, nor did they take account of other reasons for prescribing, such as patient demand. Our doctors identified a reasonable proportion of patients with psychological and social problems but were largely unaware of menstrual problems. Despite the fact that these women had a relatively high rate of psychological disturbance as measured by self report scales, no clear differences in psychological or menstrual status were found between women with and without infection, nor did the general practitioners seem to utilise such informa- 
tion directly in their decision making. They were just as likely to predict a positive result of urine analysis and prescribe antibiotics whether or not the women had detectable psychological problems. Hence we cannot confirm that psychosomatic factors were important in decision making by the doctors.

Complaints of increased frequency of micturition and dysuria were not helpful in management, perhaps because they were so common. The only presenting symptom recorded by the doctors that was significantly more common among the women with positive results was haematuria. Although this did not seem to influence the doctors' decision making, small numbers mean that we cannot rule out the possibility. Perhaps surprisingly, nocturia was not reported by the doctors as a presenting symptom. Others have reported that this pattern of frequency is common in those women who later prove to have clinically important infection. ${ }^{919}$ Possibly our doctors did not inquire as this question was left open in format.

Although we examined the patients and their management by the family practitioner in some detail, the limited size and location of the study mean that our results may not be representative of all such general practice consultations. Nevertheless, our results suggest that when women present with urinary tract symptoms, family practitioners make no particular use of physical, psychological, or menstrual factors in making their predictions but are most accurate when they have a good general knowledge of the patient. They tend to be more conservative in their management of older women and those whom they know less well.

We thank the general practitioners and patients who took part.
1 Brooke JB, Rector AL, Sheldon MG. A review of studies of decision making in general practice. Medical Information 1984;9:45-53.

2 Raynes NV. A preliminary search of procedures and patient management techniques in general practice. $\mathcal{F} R$ Coll Gen Pract 1980;30:166-72

3 Howie JGR. Clinical judgment and antibiotic use in general practice. $B M \mathcal{Y}$ 1976;ii:1061-4.

4 Royal College of General Practitioners, Office of Population Census and Surveys, Department of Health and Social Security. Morbidity statistics from general practice. Third national study 1981-82. London: HMSO, 1986:159.

5 Gaymans R, Valkenberg HA, Havekorn MJ, Gosling WRO. A prospective study of urinary tract infections in a Dutch general practice. Lancet 1976;ii:674-7.

6 Brooks D. A general practitioner's view of laboratory examination of urine. In Meers PD, ed. The bacteriological examination of urine: report of a workshop on needs and methods. London: HMSO, 1978:39-44.

7 Mond NC, Percival A, Williams JD, Brumfitt W. Presentation, diagnosis and treatment of urinary tract infection in general practice. Lancet 1965;ii:514-6.

Anderson JE. Inital treatment decisions in urinary tract infections. Canadian Family Physician 1981;27:1909-12.

9 O'Dowd T, Smail JE, West RR. Clinical judgment in the diagnosis and management of frequency and dysuria in general practice. $B M \mathcal{F} 1984 ; 288$ : $1347-9$

10 Goldberg DP. Detection of psychiatric illness by questionnaire. Oxford: Oxford University Press, 1972.

11 Clare AW, Wiggins RD. The construction of a modified version of the menstrual distress questionnaire for use in general practice populations. In: Carenza L, Zichella L. Emotions and reproduction. Vol 20A. London: Academic Press, 1979:177-84.

12 Moos RH. The development of a menstrual distress questionnaire. Psychosom Med 1968;30:853-67.

13 Moos RH. Typology of menstrual cycle symptoms. Am 7 Obstet Gynecol 1969;103:390-402.

14 Can Kasstigation beat the truth out of the urethral syndrome? Lancet 1982:ii:694-5.

15 Altman DG. Practical statistics for medical research. London: Chapman and Hall, 1991,

16 Goldthorpe $\mathrm{JH}$, Hope $\mathrm{K}$. The social grading of occupations. A new approach and scale. Oxford: Clarendon Press, 1974.

17 Hjortdahl P, Borchgrevink CF. Continuity of care: influence of general practitioners knowledge about their patients on the use of resources in consultations. BMF 1991;303:1181-4

18 Bradley CP. Uncomfortable prescribing decisions: a critical incident study. $B M 7$ 1992;304:294-6.

19 Dobbs FF, Fleming D. A simple scoring system for evaluating the symptoms, history and urine dipstick testing in the diagnosis of urinary tract infection. f $R$ Coll Gen Pract 1987;37:100-4

(Accepted 11 February 1993)

\section{A PAPER THAT CHANGED MY VIEW OF MEDICINE}

\section{The bitter sweet journey into quality}

When Donald Irvine, as chairman of the council of the Royal College of General Practitioners, published a paper in the college journal in 1983 entitled "Quality: our outstanding problem" I was innocent of the background and excited by the prospect.' When the silver tongued Irvine addressed a meeting in Nottingham on the college's new quality initiative I was spell bound.

Quality assurance had always had a minority following in academia, especially in America, but in 1983 the concepts of audit were alien to most general practitioners, myself included. When the college council approved this paper it proposed that within the next 10 years we should be able "to say, at any moment in time, what the content of our work is and what services each of us provide; and to incorporate standard setting and performance review as an integral and effective part of our professional lives."

The magnitude of this challenge cannot be underestimated. When I joined the council a year later I found that the quality initiative had largely ossified into an annual report of audits from council members. While my practice started audit ing in a tentative way I began to listen to heady talk of rellowship by assessment and higher professional training. But quality was not, seemingly, yet part of the agenda for most practitioners.

By the time that fellowship by assessment was in place my practice was ready to apply and three partners went through in the first cohort. The practice has now built in a system of continual and repetitive auditing of the conventional type, and a two monthly cycle of significant event auditing. Some of this change might have occurred without the fine words and passionate leadership of Donald Irvine, but it would never have been so profound or so sustained.

A direct lineage can be traced from the quality initiative through to medical audit and medical audit advisory groups. Without the leadership of the college it is doubtful if the cultural climate would have developed in which medical audit was the only widely welcomed part of the
NHS reforms. ${ }^{2}$ That the practice of audit has not yet met with disenchantment throughout primary care is a reflection of the fact that most general practitioners intuitively believe in Irvine's objectives.

There have, however, been side effects to the quality initiative. In its clarion call can be heard the notes that have led to the eclipse of the RCGP. It was the underlying belief that quality was measurable that led, remorselessly and logically, to the idea of rewarding good practice and the good practice allowance of the government's proposals. ${ }^{3}$ All the venom heaped on the college through the reforms and the new contract can be traced to this concept, and yet it is something that I still hold dear.

It seems to be axiomatic that the greatest improvement in standards of care come with rewards. Some rewards are deeply emotional and internal-the satisfaction of knowing a job is well done and appreciated. Some are more tangible, like attracting more patients and earning more money. That I believed that quality should be linked to tangible rewards was both logical and politically naive.

So the quality initiative inspired me and my practice and altered my professional life by making quality assurance one of my guiding lights. But it also taught me the risks of leadership and the political vulnerability of powerful ideas. Above all, the quality initiative has been a profound learning experience which I would not have missed for the world.-MIKE PRINGLE is a senior lecturer in general practice in Nottingham.

1 Irvine DH. Quality of care in general practice: our outstanding problem. $f R$ Coll Gen Pract 1983;33:521-3.

2 Secretaries of State for Health, Wales, Northern Ireland, and Scotland. Working for patients. London: HMSO, 1989.

3 Secretaries of State for Social Services, Wales, Northern Ireland, and Scotland. Primary health care-an agenda for discussion. London: HMSO, 1986.

We welcome contributions to fillers: $A$ patient who changed my practice; $A$ paper that changed my practice; The message I would most like to leave behind; $A$ memorable patient, or similar topics. 